\title{
Some remarks on Vainikko integral operators in BV type spaces
}

\author{
Laura Angeloni $^{1}$ (D) Jürgen Appell ${ }^{2} \cdot$ Simon Reinwand ${ }^{3}$
}

Ricordando Mimmo, collega e amico, con affetto, stima e gratitudine

Received: 31 May 2020 / Accepted: 10 July 2020 / Published online: 23 July 2020

(c) The Author(s) 2020

\begin{abstract}
In this paper we study Vainikko integral operators which are similar to so-called cordial integral operators and contain the classical Hardy operator, the Schur operator, and the Hilbert transform as special cases. For such operators we obtain norm estimates and equalities, mainly in BV type spaces in the sense of Jordan, Wiener, Riesz, and Waterman. Several examples are also discussed.
\end{abstract}

Keywords Cordial integral operator · Vainikko integral operator $\cdot$ BV type space $\cdot$ Norm estimate

Mathematics Subject Classification $47 \mathrm{G} 10 \cdot 26 \mathrm{~A} 45 \cdot 45 \mathrm{D} 05 \cdot 45 \mathrm{H} 05 \cdot 45 \mathrm{P} 05$

The first author is a member of the group GNAMPA of the Istituto Nazionale di Alta Matematica (INdAM) and of the "Research Italian Network on Approximation"; she was partially supported by Department of Mathematics and Computer Science of the University of Perugia (Italy), by the project "Integrazione, Approssimazione, Analisi Nonlineare e loro Applicazioni", funded by the 2019 basic research fund of the University of Perugia and by the 2020 GNAMPA project "Metodi di analisi reale e di teoria della misura per l'approssimazione attraverso operatori discreti e applicazioni".

$\bowtie \quad$ Laura Angeloni

laura.angeloni@unipg.it

Jürgen Appell

jurgen@dmuw.de

Simon Reinwand

sreinwand@dmuw.de

1 Department of Mathematics and Computer Science, University of Perugia, Via Vanvitelli 1, 06123 Perugia, Italy

2 Department of Mathematics, University of Würzburg, Emil-Fischer-Str. 30, 97074 Würzburg, Germany

3 Department of Mathematics, University of Würzburg, Emil-Fischer-Str. 40, 97074 Würzburg, Germany 


\section{Introduction}

Domenico Candeloro (called "Mimmo" by his friends) was one of the leading specialists in the theory, methods, and applications of integral operators. He has given many important contributions to this field, with a particular emphasis on exotic measures, non-standard integrals, and multivalued maps. In the list of references at the end we mention only the more recent papers [2-25] he wrote, in part with coauthors, in the last 20 years.

In this paper we study a class of integral operators in a much simpler setting, using only single-valued scalar functions and integrals defined by the classical Lebesgue measure on the real line. In spite of their simplicity, we are convinced that our results on mapping properties of such integral operators would have been appreciated by Mimmo. In our discussion we will give particular attention to spaces of functions of bounded variation, a topic that is also very much en vogue in the Analysis School of the University of Perugia which owes so much to Mimmo's scientific activity.

\section{Cordial integral operators}

Given a nonnegative $L_{1}$ function $\varphi:(0,1) \rightarrow \mathbb{R}$, in [27] the author defines an associated Volterra integral operator $V_{\varphi}$ by

$$
\left(V_{\varphi} x\right)(t)=\frac{1}{t} \int_{0}^{t} \varphi\left(\frac{s}{t}\right) x(s) d s=\int_{0}^{1} \varphi(\tau) x(t \tau) d \tau .
$$

Such operators are called cordial, the generating function $\varphi$ the core of $V_{\varphi}$. In [27] and [28] the author gives necessary and sufficient conditions for $V_{\varphi}$ to be bounded in the spaces $C$, $C^{m}$ and $L_{\infty}$. In the recent paper [29] he develops a parallel theory for Lebesgue spaces and proves that $V_{\varphi}$ is bounded in $L_{p}(1 \leq p \leq \infty)$ iff

$$
\int_{0}^{1} s^{-1 / p}|\varphi(s)| d s<\infty
$$

Moreover, he shows that in this case the norm $\left\|V_{\varphi}\right\|_{L_{p} \rightarrow L_{p}}$ coincides with this integral. The spectrum and essential spectrum of the operator are also calculated.

Cordial integral operators have a series of remarkable properties. For example, one may show that the operator (1) has the continuum of eigenfunctions $u_{r}(t)=t^{r}(0 \leq r<\infty)$ in the space $C$, and so it cannot be compact. Moreover, the eigenvalues $\lambda$ satisfy $\left\|\lambda I-V_{\varphi}\right\|_{C \rightarrow C}=$ $|\lambda|+\|\varphi\|_{L_{1}} ;$ in particular, $\left\|V_{\varphi}\right\|_{C \rightarrow C}=\|\varphi\|_{L_{1}}$.

Interestingly, a certain converse is also true: if the general Volterra operator

$$
(V x)(t)=\int_{0}^{t} k(t, s) x(s) d s
$$

has the continuum of eigenfunctions $u_{r}(t)=t^{r}(0 \leq r<\infty)$, and its kernel function $k:[0,1] \times[0,1] \rightarrow \mathbb{R}$ satisfies some regularity requirements, then $V=V_{\varphi}$ for $\varphi:=$ $k(1, \cdot) \in L_{1}$. 


\section{Vainikko integral operators in Lebesgue spaces}

In this section we slightly modify the definition (1) to cover a wider range of examples. Given an $L_{1}$ function $\varphi:(0, \infty) \rightarrow \mathbb{R}$, let us call the operator $V_{\varphi}$ defined by

$$
\left(V_{\varphi} x\right)(t)=\frac{1}{t} \int_{0}^{\infty} \varphi\left(\frac{s}{t}\right) x(s) d s=\int_{0}^{\infty} \varphi(\tau) x(t \tau) d \tau
$$

Vainikko integral operator in the sequel. So the only difference between cordial and Vainikko operators is that we extend the integration in (2) over the semiaxis $(0, \infty)$. Prominent examples for such operators are the Hardy operator

$$
(H x)(t)=\frac{1}{t} \int_{0}^{t} x(s) d s
$$

which has the form (2) for the choice $\varphi(s):=\chi_{(0,1]}(s)$, the Schur operator

$$
(S x)(t)=\int_{0}^{\infty} \frac{x(s)}{\max \{s, t\}} d s
$$

which has the form (2) for the choice $\varphi(s):=1 / \max \{1, s\}$, and the (strongly singular) Hilbert transform

$$
(T x)(t)=\int_{0}^{\infty} \frac{x(s)}{s+t} d s
$$

which has the form (2) for the choice $\varphi(s):=1 /(1+s)$.

Our first result gives a two-sided estimate for the norm of a Vainikko operator in the Lebesgue space $L_{p}[0, \infty)$. To this end, we will use the shortcut

$$
\varphi_{\theta}(s):=s^{\theta} \varphi(s) \quad(s>0),
$$

where $|\theta| \leq 1$.

Theorem 3.1 For $1<p<\infty$, suppose that the Vainikko integral operator $V_{\varphi}$ defined by (2) maps $L_{p}[0, \infty)$ into itself. Let $\varphi:[0, \infty) \rightarrow \mathbb{R}$ be measurable with the property that

$$
\left\|\varphi_{-1 / p}\right\|_{L_{1}}<\infty .
$$

Then $V_{\varphi}$ is bounded and satisfies the estimate

$$
\left|\int_{0}^{\infty} s^{-1 / p} \varphi(s) d s\right| \leq\left\|V_{\varphi}\right\|_{L_{p} \rightarrow L_{p}} \leq \int_{0}^{\infty} s^{-1 / p}|\varphi(s)| d s .
$$

Consequently, in case $\varphi \geq 0$ or $\varphi \leq 0$, the norm $\left\|V_{\varphi}\right\|_{L_{p} \rightarrow L_{p}}$ of $V_{\varphi}$ coincides with the right-hand side of (7).

Proof Although the proof may be found in [29] for $L_{p}[0,1]$, we give here another proof which requires slightly different arguments, since $L_{p}(I)$ is not included in $L_{q}(I)$ for $q \leq p$ in case of an unbounded interval $I$. To estimate the norm $\left\|V_{\varphi}\right\|_{L_{p} \rightarrow L_{p}}$, we use the fact that the bilinear form

$$
\langle y, z\rangle:=\int_{0}^{\infty} y(t) z(t) d t \quad\left(y \in L_{p}, z \in L_{p^{\prime}}\right)
$$

establishes a duality between $L_{p}$ and $L_{p^{\prime}}$ for $p^{\prime}:=p /(p-1)$, in the sense that

$$
\|y\|_{L_{p}}=\sup \left\{\langle y, z\rangle:\|z\|_{L_{p^{\prime}}} \leq 1\right\}, \quad\|z\|_{L_{p^{\prime}}}=\sup \left\{\langle y, z\rangle:\|y\|_{L_{p}} \leq 1\right\} .
$$


For $x \in L_{p}$ and $y:=V_{\varphi} x$ we get, by Fubini's theorem

$$
\begin{aligned}
\langle y, z\rangle= & \int_{0}^{\infty}\left(\frac{1}{t} \int_{0}^{\infty} \varphi\left(\frac{s}{t}\right) x(s) d s\right) z(t) d t=\int_{Q} \frac{1}{t} \varphi\left(\frac{s}{t}\right) x(s) z(t) d t d s \\
= & \int_{Q}\left\{\left(\frac{s}{t}\right)^{(p-1) / p^{2}}\left[\frac{1}{t} \varphi\left(\frac{s}{t}\right)\right]^{1 / p} x(s)\right\} \\
& \times\left\{\left(\frac{t}{s}\right)^{(p-1) / p^{2}}\left[\frac{1}{t} \varphi\left(\frac{s}{t}\right)\right]^{1 / p^{\prime}} z(t)\right\} d t d s,
\end{aligned}
$$

where $Q:=(0, \infty) \times(0, \infty)$. Therefore Hölder's inequality implies

$$
\begin{aligned}
\left|\left\langle V_{\varphi} x, z\right\rangle\right| \leq & \left\{\int_{Q}\left(\frac{s}{t}\right)^{1-1 / p}\left|\varphi\left(\frac{s}{t}\right)\right||x(s)|^{p} \frac{d t}{t} d s\right\}^{1 / p} \\
& \times\left\{\int_{Q}\left(\frac{t}{s}\right)^{1 / p}\left|\varphi\left(\frac{s}{t}\right)\right||z(t)|^{p^{\prime}} \frac{d t}{t} d s\right\}^{1 / p^{\prime}} .
\end{aligned}
$$

Using the change of variables $s:=t \tau$ we get

$$
\int_{0}^{\infty}\left(\frac{s}{t}\right)^{1-1 / p}\left|\varphi\left(\frac{s}{t}\right)\right| \frac{d t}{t}=\int_{0}^{\infty} \tau^{-1 / p}|\varphi(\tau)| d \tau,
$$

and applying again Fubini's theorem we conclude that the first integral in (8) is

$$
\int_{Q}\left(\frac{s}{t}\right)^{1-1 / p}\left|\varphi\left(\frac{s}{t}\right)\right||x(s)|^{p} \frac{d t}{t} d s=\left(\int_{0}^{\infty} \tau^{-1 / p}|\varphi(\tau)| d \tau\right)\left(\int_{0}^{\infty}|x(s)|^{p} d s\right) .
$$

A similar calculation gives

$$
\int_{Q}\left(\frac{t}{s}\right)^{1 / p}\left|\varphi\left(\frac{s}{t}\right)\right||z(t)|^{p^{\prime}} \frac{d t}{t} d s=\left(\int_{0}^{\infty} \tau^{-1 / p}|\varphi(\tau)| d \tau\right)\left(\int_{0}^{\infty}|z(t)|^{p^{\prime}} d t\right)
$$

for the second integral in (8). Combining these equalities we end up with

$$
\left|\left\langle V_{\varphi} x, z\right\rangle\right| \leq\|x\|_{L_{p}}\|z\|_{L_{p^{\prime}}}\left(\int_{0}^{\infty} \tau^{-1 / p}|\varphi(\tau)| d \tau\right)^{1 / p}\left(\int_{0}^{\infty} \tau^{-1 / p}|\varphi(\tau)| d \tau\right)^{1 / p^{\prime}}
$$

which in view of $1 / p+1 / p^{\prime}=1$ proves the claim.

To illustrate Theorem 3.1 let us go back to our examples mentioned above.

Example 3.1 For $\varphi(s):=\chi_{(0,1]}(s)$ we have

$$
\left\|\varphi_{-1 / p}\right\|_{L_{1}}=\int_{0}^{\infty} s^{-1 / p} \varphi(s) d s=\int_{0}^{1} s^{-1 / p} d s=\frac{p}{p-1}
$$

which gives the precise norm $\|H\|_{L_{p} \rightarrow L_{p}}$ of the Hardy operator (3) in $L_{p}$; in particular, $\|H\|_{L_{2} \rightarrow L_{2}}=2$. Similarly, for $\varphi(s):=1 / \max \{1, s\}$ we have

$$
\left\|\varphi_{-1 / p}\right\|_{L_{1}}=\int_{0}^{\infty} s^{-1 / p} \varphi(s) d s=\int_{0}^{1} s^{-1 / p} d s+\int_{1}^{\infty} s^{-1-1 / p} d s=\frac{p^{2}}{p-1}
$$


which gives the precise norm $\|S\|_{L_{p} \rightarrow L_{p}}$ of the Schur operator (4) in $L_{p}$; in particular, $\|S\|_{L_{2} \rightarrow L_{2}}=4$. Finally, for $\varphi(s):=1 /(1+s)$ we have

$$
\left\|\varphi_{-1 / p}\right\|_{L_{1}}=\int_{0}^{\infty} s^{-1 / p} \varphi(s) d s=\int_{0}^{\infty} \frac{s^{-1 / p}}{1+s} d s=\frac{\pi}{\sin (\pi / p)}
$$

which gives the precise norm $\|T\|_{L_{p} \rightarrow L_{p}}$ of the Hilbert transform (5) in $L_{p}$; in particular, $\|T\|_{L_{2} \rightarrow L_{2}}=\pi$.

The question arises whether or not it is possible to extend Theorem 3.1 to the extreme cases $p=1$ or $p=\infty$. Here, the norm equalities in Theorem 3.1 illustrate the difference between the operator (3), on the one hand, and the operators (4) and (5), on the other. Since the last expression in (9) tends to 1 as $p \rightarrow \infty$, say, one might hope that the Hardy operator also maps $L_{\infty}$ into itself with $\|H\|_{L_{\infty} \rightarrow L_{\infty}}=1$; this may be in fact verified by a simple calculation. On the other hand, since the last expression in (10) or (11) tends to $\infty$ as $p \rightarrow \infty$, one might suspect that the Schur operator and the Hilbert transform do not map $L_{\infty}$ into itself. Indeed, for $x(t) \equiv 1$ we get

$$
(S x)(t)=\int_{0}^{\infty} \frac{d s}{\max \{s, t\}}=1+\int_{t}^{\infty} \frac{d s}{s}, \quad(T x)(t)=\int_{0}^{\infty} \frac{d s}{s+t}=\int_{t}^{\infty} \frac{d s}{s},
$$

and so $S x \notin L_{\infty}$ and $T x \notin L_{\infty}$.

\section{Vainikko integral operators in Wiener spaces}

In view of the importance of integral operators in spaces of functions of bounded (classical or generalized) variation, it seems reasonable to study the operator (2) in the space $B V$ and its various generalizations. This is the purpose of this section. In contrast to $L_{p}$-spaces, however, $B V$-type spaces have a reasonable norm only for functions on compact intervals, but not on the semiaxis $[0, \infty)$. Since our main emphasis is on norm estimates in this paper, in what follows we consider functions $x:[0,1] \rightarrow \mathbb{R}$. In this case for norm estimates we will use the fact that

$$
\left\|\varphi_{\theta}\right\|_{L_{1}} \leq\|\varphi\|_{L_{1}}, \quad\left|\left(V_{\varphi} x\right)(0)\right|=\left|\int_{0}^{1} \varphi(\tau) x(0) d \tau\right| \leq\|\varphi\|_{L_{1}}|x(0)| .
$$

Given $p \in[1, \infty)$ and a partition $P:=\left\{t_{0}, t_{1}, \ldots, t_{m-1}, t_{m}\right\}$ of $[0,1]$, we denote by

$$
\operatorname{Var}_{p}(x, P ;[0,1]):=\sum_{j=1}^{m}\left|x\left(t_{j}\right)-x\left(t_{j-1}\right)\right|^{p}
$$

the Wiener $p$-variation of $x:[0,1] \rightarrow \mathbb{R}$ w.r.t. $P$, and by

$$
\operatorname{Var}_{p}(x)=\operatorname{Var}_{p}(x ;[0,1]):=\sup _{P} \operatorname{Var}_{p}(x, P ;[0,1])
$$

its total Wiener $p$-variation on $[0,1]$. In case $\operatorname{Var}_{p}(x ;[0,1])<\infty$ we write $x \in B V_{p}[0,1]$. The set $B V_{p}[0,1]$ equipped with the norm

$$
\|x\|_{B V_{p}}:=|x(0)|+\operatorname{Var}_{p}(x ;[0,1])^{1 / p}
$$


is a Banach space [32]. A particular important special case is of course $p=1$, where we obtain the classical Jordan variation

$$
\operatorname{Var}(x)=\operatorname{Var}(x ;[0,1]):=\sup _{P} \operatorname{Var}(x, P ;[0,1])=\sup _{P} \sum_{j=1}^{m}\left|x\left(t_{j}\right)-x\left(t_{j-1}\right)\right|
$$

and the space $B V_{1}[0,1]=B V[0,1]$ equipped with the norm $\|x\|_{B V}:=|x(0)|+$ $\operatorname{Var}(x ;[0,1])$.

Theorem 4.1 In case $\varphi \in L_{p}$ the operator $V_{\varphi}$ is bounded in $B V_{p}$ and satisfies the estimates

$$
\left|\int_{0}^{1} \varphi(s) d s\right| \leq\left\|V_{\varphi}\right\|_{B V_{p} \rightarrow B V_{p}} \leq\|\varphi\|_{L_{p}} .
$$

Proof Fix $x \in B V_{p}$ and a partition $P:=\left\{t_{0}, t_{1}, \ldots, t_{m-1}, t_{m}\right\}$ of $[0,1]$. Then we get, by Jensen's inequality,

$$
\begin{aligned}
\sum_{j=1}^{m}\left|\left(V_{\varphi} x\right)\left(t_{j}\right)-\left(V_{\varphi} x\right)\left(t_{j-1}\right)\right|^{p} & =\sum_{j=1}^{m}\left|\int_{0}^{1} \varphi(s)\left[x\left(s t_{j}\right)-x\left(s t_{j-1}\right)\right] d s\right|^{p} \\
& \leq \int_{0}^{1}|\varphi(s)|^{p} \sum_{j=1}^{m}\left|x\left(s t_{j}\right)-x\left(s t_{j-1}\right)\right|^{p} d s \leq\|\varphi\|_{L_{p}}^{p} \operatorname{Var}_{p}(x) .
\end{aligned}
$$

Combining this estimate with (12) we conclude that $\left\|V_{\varphi} x\right\|_{B V_{p}} \leq\|\varphi\|_{L_{p}}\|x\|_{B V_{p}}$, which proves the upper estimate in (13).

For the proof of the lower estimate it suffices to take $e(t) \equiv 1$ and to note that, for all $p \geq 1,\|e\|_{B V_{p}}=1,\left\|V_{\varphi} e\right\|_{B V_{p}}=\left|\int_{0}^{1} \varphi(s) d s\right|$.

Since $B V_{1}=B V$, we get the estimate

$$
\left|\int_{0}^{1} \varphi(s) d s\right| \leq\left\|V_{\varphi}\right\|_{B V \rightarrow B V} \leq \int_{0}^{1}|\varphi(t)| d s
$$

as a special case of (13). Consequently, in case $\varphi \geq 0$ or $\varphi \leq 0$ a.e. on $[0,1]$ the norm $\left\|V_{\varphi}\right\|_{B V \rightarrow B V}$ coincides with the $L_{1}$-norm of $\varphi$. It is not clear whether or not this is also true if $\varphi$ changes its sign on subsets of positive measure. However, the lower and upper bounds in the estimates (13) may drift apart the more "symmetric" $\varphi$ changes sign and has large absolute values, as the following example suggests.

Example 4.1 For $c>0$, consider the bang-bang function $\psi_{c}:[0,1] \rightarrow \mathbb{R}$ defined by $\psi_{c}(t):=c \chi_{[0,1 / 2]}(t)-c \chi_{(1 / 2,1]}(t)$. Clearly, $\left\|\psi_{c}\right\|_{L_{p}}=c$ for all $p \in[1, \infty]$, and $\int_{0}^{1} \psi_{c}(t) d t=0$. Moreover, it is not hard to see that $\psi_{c} \in B V_{p}$ for all $p \geq 1$ with

$$
\left\|\psi_{c}\right\|_{B V_{p}}=\left|\psi_{c}(0)\right|+\operatorname{Var}_{p}\left(\psi_{c} ;[0,1]\right)^{1 / p}=3 c .
$$

So we may apply the operator $V_{\psi_{c}}$ to $\psi_{c}$ itself and obtain, by (1),

$$
\left(V_{\psi_{c}} \psi_{c}\right)(t)=\int_{0}^{1} \psi_{c}(\tau) \psi_{c}(t \tau) d \tau= \begin{cases}0 & \text { for } 0 \leq t \leq 1 / 2, \\ c^{2}(2-1 / t) & \text { for } 1 / 2<t \leq 1\end{cases}
$$

This implies that

$$
\left\|V_{\psi_{c}} \psi_{c}\right\|_{B V_{p}}=\left|V_{\psi_{c}} \psi_{c}(0)\right|+\operatorname{Var}_{p}\left(V_{\psi_{c}} \psi_{c} ;[0,1]\right)^{1 / p}=c^{2} .
$$

Thus, a comparison of (15) and (16) shows that $\left\|V_{\psi_{c}}\right\|_{B V_{p} \rightarrow B V_{p}} \geq c / 3$. We conclude that the lower bound in (13) is 0 , while the upper bound is $c$ which may become arbitrarily large. 


\section{Vainikko integral operators in Riesz spaces}

Another generalization of the space $B V$ is due to Riesz [26]. Given $p \in[1, \infty)$ and a partition $P:=\left\{t_{0}, t_{1}, \ldots, t_{m-1}, t_{m}\right\}$ of $[0,1]$ as before, we denote by

$$
R \operatorname{Var}_{p}(x, P ;[0,1]):=\sum_{j=1}^{m} \frac{\left|x\left(t_{j}\right)-x\left(t_{j-1}\right)\right|^{p}}{\left(t_{j}-t_{j-1}\right)^{p-1}}
$$

the Riesz $p$-variation of a function $x$ w.r.t. $P$. The total Riesz $p$-variation of $x:[0,1] \rightarrow \mathbb{R}$ on $[0,1]$ is then given by

$$
R \operatorname{Var}_{p}(x)=R \operatorname{Var}_{p}(x ;[0,1]):=\sup _{P} \operatorname{RVar}_{p}(x, P ;[0,1]) .
$$

In case $R \operatorname{Var}_{p}(x ;[0,1])<\infty$ we write $x \in R B V_{p}[0,1]$. The set $R B V_{p}[0,1]$ equipped with the norm

$$
\|x\|_{R B V_{p}}:=|x(0)|+R \operatorname{Var}_{p}(x ;[0,1])^{1 / p}
$$

is a Banach space [26]. In spite of their similarity, the spaces $B V_{p}$ and $R B V_{p}$ have quite different properties. First of all, the scale of spaces $B V_{p}$ is increasing in $p$, while the scale of spaces $R B V_{p}$ is decreasing in $p$. Moreover, every function in the Riesz space $R B V_{p}$ is continuous for $p>1$, but $R B V_{1}=B V$ contains of course many discontinuous functions. However, the most interesting property of Riesz spaces is that, for $1<p<\infty$, from $x \in R B V_{p}$ it follows that $x$ is absolutely continuous with $x^{\prime} \in L_{p}$ and $R \operatorname{Var}_{p}(x)=\left\|x^{\prime}\right\|_{L_{p}}^{p}$, and vice versa [1]. This means that Riesz discovered Sobolev spaces, at least in the scalar case, 20 years before Sobolev. This fact allows us to use Theorem 3.1 for finding a condition for $V_{\varphi}$ to map $R B V_{p}$ into itself.

Theorem 5.1 In case $\varphi \in L_{1}$ the operator $V_{\varphi}$ is bounded in $R B V_{p}$ and satisfies the estimates

$$
\left|\int_{0}^{1} \varphi(s) d s\right| \leq\left\|V_{\varphi}\right\|_{R B V_{p} \rightarrow R B V_{p}} \leq\|\varphi\|_{L_{1}} .
$$

Consequently, in case $\varphi \geq 0$ or $\varphi \leq 0$, the norm $\left\|V_{\varphi}\right\|_{R B V_{p} \rightarrow R B V_{p}}$ of $V_{\varphi}$ coincides with the right-hand side of (17).

Proof The case $p=1$ is covered by (14), so let $1<p<\infty$. In this case, $x$ is absolutely continuous and $x^{\prime} \in L_{p}$. Moreover, using the second integral in (2) we see that

$$
\left(V_{\varphi} x\right)^{\prime}(t)=\int_{0}^{1} \varphi(\tau) \tau x^{\prime}(t \tau) d \tau=\left(V_{\varphi_{1}} x^{\prime}\right)(t)
$$

where we have used the notation (6). From $\varphi \in L_{1}$ it follows that also $\varphi_{1-1 / p} \in L_{1}$; so Vainikko's result implies that $V_{\varphi}$ maps $R B V_{p}$ into itself and, by (18),

$$
\begin{aligned}
R \operatorname{Var}_{p}\left(V_{\varphi} x\right) & =\left\|V_{\varphi_{1}} x^{\prime}\right\|_{L_{p}}^{p} \leq\left\|V_{\varphi_{1}}\right\|_{L_{p} \rightarrow L_{p}}^{p}\left\|x^{\prime}\right\|_{L_{p}}^{p} \leq\left\|\varphi_{1-1 / p}\right\|_{L_{1}}^{p} R \operatorname{Var}_{p}(x) \\
& \leq\|\varphi\|_{L_{1}}^{p} R \operatorname{Var}_{p}(x) .
\end{aligned}
$$

So together with (12) this yields $\left\|V_{\varphi} x\right\|_{R B V_{p}} \leq\left|\left(V_{\varphi} x\right)(0)\right|+R \operatorname{Var}_{p}\left(V_{\varphi} x\right)^{1 / p} \leq$ $\|\varphi\|_{L_{1}}\|x\|_{R B V_{p}}$.

The lower estimate in (17) is proved exactly as before. 
Observe that $R B V_{\infty}=L i p$, the linear space of all Lipschitz continuous maps with norm $\|x\|_{\text {Lip }}=|x(0)|+\operatorname{lip}(x ;[0,1])$, where $\operatorname{lip}(x ;[0,1]):=\sup \left\{\frac{|x(s)-x(t)|}{|s-t|}: 0 \leq s, t \leq 1\right.$, $s \neq t\}$. So we get in addition as a fringe benefit from Theorem 5.1 the following

Theorem 5.2 The operator $V_{\varphi}$ is bounded in Lip iff $\varphi_{1} \in L_{1}$. In this case we have

$$
\left|\int_{0}^{1} \varphi(s) d s\right| \leq\left\|V_{\varphi}\right\|_{L i p \rightarrow \text { Lip }} \leq\|\varphi\|_{L_{1}} .
$$

Consequently, in case $\varphi \geq 0$ or $\varphi \leq 0$, the norm $\left\|V_{\varphi}\right\|_{\text {Lip } \rightarrow \text { Lip }}$ of $V_{\varphi}$ coincides with $\|\varphi\|_{L_{1}}$.

Observe that this result for the space Lip may be easily proved directly. In fact, from $|x(s)-x(t)| \leq L|s-t|$ it follows that $\left|\left(V_{\varphi} x\right)(s)-\left(V_{\varphi} x\right)(t)\right| \leq \int_{0}^{1} \varphi(\tau)|x(\tau s)-x(\tau t)| d \tau \leq$ $L\left\|\varphi_{1}\right\|_{L_{1}}|s-t|$, hence $\operatorname{lip}\left(V_{\varphi} x\right) \leq\left\|\varphi_{1}\right\|_{L_{1}} \operatorname{lip}(x) \leq\|\varphi\|_{L_{1}} \operatorname{lip}(x)$.

Let us illustrate our results by means of our "test animals", the Hardy operator (3), the Schur operator (4), and the Hilbert transform (5).

For $\varphi(s):=\chi_{(0,1]}(s)$ we have $\|\varphi\|_{L_{p}}^{p}=\int_{0}^{\infty} \chi_{(0,1]}(s)^{p} d s \equiv 1$ and $\left\|\varphi_{1-1 / p}\right\|_{L_{1}}=$ $\int_{0}^{\infty} s^{1-1 / p} \chi_{(0,1]}(s) d s=\int_{0}^{1} s^{1-1 / p} d s<\infty$ for any $p \geq 1$. So the Hardy operator is bounded in $B V_{p}$ for $1 \leq p<\infty$ and in $R B V_{p}$ for $1 \leq p \leq \infty$.

On the other hand, for $\varphi(s):=1 / \max \{1, s\}$ we have $\|\varphi\|_{L_{p}}^{p}=\int_{0}^{1} 1 d s+\int_{1}^{\infty} s^{-p} d s<\infty$ for $p>1$, and $\left\|\varphi_{1-1 / p}\right\|_{L_{1}}=\int_{0}^{1} s^{1-1 / p} d s+\int_{1}^{\infty} s^{-1 / p} d s=\infty$. So the Schur operator maps the space $B V_{p}$ for $p>1$ into itself, but none of the spaces $R B V_{p}$.

Finally, for $\varphi(s):=1 /(1+s)$ we have $\|\varphi\|_{L_{p}}^{p}=\int_{0}^{\infty} \frac{d s}{(1+s)^{p}}=\int_{1}^{\infty} \frac{d s}{s^{p}}<\infty$ for $p>1$, and $\left\|\varphi_{1-1 / p}\right\|_{L_{1}}=\int_{0}^{\infty} \frac{s^{1-1 / p}}{1+s} d s=\infty$ for $p>1$. So for $p>1$ the Hilbert transform maps the space $B V_{p}$ into itself, but not the space $R B V_{p}$.

We close this section with another operator which depends on a real parameter $\alpha$ and is, in contrast to the Hilbert transform, weakly singular.

Example 5.1 For $\alpha>0$, consider the Liouville operator

$$
\left(L_{\alpha} x\right)(t)=\frac{1}{t^{\alpha}} \int_{0}^{t} s^{\alpha-1} x(s) d s .
$$

This operator has the form (2) for the choice $\varphi(s):=s^{\alpha-1} \chi_{(0,1]}(s)$. Since $\left\|\varphi_{-1 / p}\right\|_{L_{1}}=$ $\int_{0}^{1} s^{-1 / p} s^{\alpha-1} d s=\frac{1}{\alpha-1 / p}$, the operator (19) maps $L_{p}$ for $p \alpha>1$ into itself. Moreover, since $\|\varphi\|_{L_{p}}^{p}=\int_{0}^{1} s^{(\alpha-1) p} d s$ and $\left\|\varphi_{1-1 / p}\right\|_{L_{1}}=\int_{0}^{1} s^{\alpha-1 / p} d s$, the Liouville operator is bounded in $B V_{p}$ for $\alpha>1-1 / p$, i.e., $p<1 /(1-\alpha)$, and in $R B V_{p}$ for every $\alpha>0$.

\section{Vainikko integral operators in Waterman spaces}

Finally, let us recall yet another $B V$-type space which was introduced by Waterman [30] and has very interesting applications.

Let $\Lambda:=\left(\lambda_{k}\right)_{k}$ be a positive decreasing sequence satisfying $\lim _{k \rightarrow \infty} \lambda_{k}=0$ and $\sum_{k=1}^{\infty} \lambda_{k}=\infty$. Recall that the Waterman variation of a function $x:[0,1] \rightarrow \mathbb{R}$ w.r.t. a collection $S=\left\{\left[a_{k}, b_{k}\right]: k=1,2, \ldots, n\right\}$ (with $n$ variable) of pairwise non-overlapping 
intervals $\left[a_{k}, b_{k}\right] \subset[0,1]$ is defined by

$$
\operatorname{Var}_{\Lambda}(x, S ;[0,1]):=\sum_{k=1}^{n} \lambda_{k}\left|x\left(b_{k}\right)-x\left(a_{k}\right)\right|,
$$

and the total Waterman variation of $x$ on $[0,1]$ by

$$
\operatorname{Var}_{\Lambda}(x ;[0,1]):=\sup _{S} \operatorname{Var}_{\Lambda}(x, S ;[0,1]) .
$$

In case $\operatorname{Var}_{\Lambda}(x ;[a, b])<\infty$ we write $x \in \Lambda B V[0,1]$. The set $\Lambda B V[0,1]$ equipped with the norm

$$
\|x\|_{\Lambda B V}:=|x(0)|+\operatorname{Var}_{\Lambda}(x ;[0,1])
$$

is a Banach space [30]. A typical example is of course $\Lambda_{q}:=\left(k^{-q}\right)_{k}$ for $0<q \leq 1$; this has important applications to Fourier series. Thus, in [31] it was shown that, for $f \in \Lambda_{q} B V$, the Fourier series of $f$ is everywhere $(C, \beta)$-bounded for $\beta=q-1$, and $(C, \alpha)$-summable for $\alpha>q-1$. Moreover, these estimates for $\alpha$ and $\beta$ are sharp. The starting point for the study of Waterman spaces was the choice $q=1$; in this case the elements of the space $\Lambda_{1} B V=: H B V$ are called functions of bounded harmonic variation.

Theorem 6.1 In case $\varphi \in L_{1}$ the operator $V_{\varphi}$ is bounded in $\Lambda B V$ and satisfies the estimates

$$
\left|\int_{0}^{1} \varphi(s) d s\right| \leq\left\|V_{\varphi}\right\|_{\Lambda B V \rightarrow \Lambda B V} \leq\|\varphi\|_{L_{1}} .
$$

Consequently, in case $\varphi \geq 0$ or $\varphi \leq 0$, the norm $\left\|V_{\varphi}\right\|_{\text {Lip } \rightarrow \text { Lip }}$ of $V_{\varphi}$ coincides with $\|\varphi\|_{L_{1}}$.

Proof Fix $x \in \Lambda B V$ and a collection $S=\left\{\left[a_{k}, b_{k}\right]: k=1,2, \ldots, n\right\}$ of non-overlapping intervals $\left[a_{k}, b_{k}\right] \subset[0,1]$. Then

$$
\begin{aligned}
\sum_{k=1}^{n} \lambda_{k}\left|\left(V_{\varphi} x\right)\left(b_{k}\right)-\left(V_{\varphi} x\right)\left(a_{k}\right)\right| & =\sum_{k=1}^{n} \lambda_{k}\left|\int_{0}^{1} \varphi(s)\left[x\left(s b_{k}\right)-x\left(s a_{k}\right)\right] d s\right| \\
& \leq \int_{0}^{1}|\varphi(s)| \sum_{k=1}^{n} \lambda_{k}\left|x\left(s b_{k}\right)-x\left(s a_{k}\right)\right| d s \leq\|\varphi\|_{L_{1}} \operatorname{Var}_{\Lambda}(x) .
\end{aligned}
$$

Adding this estimate to (12) we see that $\left\|V_{\varphi} x\right\|_{\Lambda B V} \leq\|\varphi\|_{L_{1}}\|x\|_{\Lambda B V}$, which proves the upper estimate in (20). For the proof of the lower estimate we take the same function $e(t) \equiv 1$ as in the proof of Theorem 4.1 .

To conclude, let us summarize our norm estimates for the most important example, the Hardy operator (3), in the following

Example 6.1 We already know that $\|H\|_{L_{p} \rightarrow L_{p}}=p /(p-1)$ for $1<p<\infty$ and $\|H\|_{L_{\infty} \rightarrow L_{\infty}}=1$. Since $\int_{0}^{1} \varphi(s) d s=1$ and $\|\varphi\|_{L_{p}}=1$ for $\varphi(s)=\chi_{(0,1]}(s)$, from Theorems 4.1, 5.1 and 6.1 we conclude that $\|H\|_{B V_{p} \rightarrow B V_{p}}=\|H\|_{R B V_{p} \rightarrow R B V_{p}}=$ $\|H\|_{\Lambda B V \rightarrow \Lambda B V}=1$.

Acknowledgements Open access funding provided by Università degli Studi di Perugia within the CRUICARE Agreement.

\section{Compliance with ethical standards}

Conflict of interest On behalf of all authors, the corresponding author states that there is no conflict of interest. 
Open Access This article is licensed under a Creative Commons Attribution 4.0 International License, which permits use, sharing, adaptation, distribution and reproduction in any medium or format, as long as you give appropriate credit to the original author(s) and the source, provide a link to the Creative Commons licence, and indicate if changes were made. The images or other third party material in this article are included in the article's Creative Commons licence, unless indicated otherwise in a credit line to the material. If material is not included in the article's Creative Commons licence and your intended use is not permitted by statutory regulation or exceeds the permitted use, you will need to obtain permission directly from the copyright holder. To view a copy of this licence, visit http://creativecommons.org/licenses/by/4.0/.

\section{References}

1. Appell, J., Bugajewska, D., Kasprzak, P., Reinwand, S.: Applications of $B V$ type spaces (submitted)

2. Boccuto, A., Candeloro, D.: Uniform $s$-boundedness and convergence results for measures with values in complete (1)-groups. J. Math. Anal. Appl. 265(1), 170-194 (2002)

3. Boccuto, A., Candeloro, D.: Vitali and Schur-type theorems for Riesz space valued set functions. Atti Sem. Mat. Fis. Univ. Modena 50(1), 85-103 (2002)

4. Boccuto, A., Candeloro, D.: Integral and differential in Riesz spaces and applications. J. Appl. Funct. Anal. 3(1), 89-111 (2008)

5. Boccuto, A., Candeloro, D.: Integral and ideals in Riesz spaces. Inf. Sci. 179(17), 2891-2902 (2009)

6. Boccuto, A., Candeloro, D., Riečan, B.: Abstract generalized Kurzweil-Henstock-type integrals for Riesz space valued functions. Real Anal. Exch. 34(1), 171-194 (2009)

7. Boccuto, A., Candeloro, D., Sambucini, A.R.: Stieltjes-type integrals for metric semigroup-valued functions defined on unbounded intervals. Panamer. Math. J. 17(4), 39-58 (2007)

8. Boccuto, A., Candeloro, D., Sambucini, A.R.: A Fubini theorem in Riesz spaces for the Kurzweil-Hnstock integral. J. Funct. Spaces Appl. 9(3), 283-304 (2011)

9. Boccuto, A., Candeloro, D., Sambucini, A.R.: Henstock multivalued integrability in Banach lattices with respect to pointwise non atomic measures. Atti Accad. Naz. Lincei Cl. Sci. Fis. Mat. Nat. 26(4), 363-383 (2015)

10. Brooks, J.K., Candeloro, D.: Weak stochastic integration in Banach spaces. Atti Sem. Mat. Fis. Univ. Modena 49(2), 513-522 (2001)

11. Brooks, J.K., Candeloro, D.: On the Caccioppoli integral. Atti Sem. Mat. Fis. Univ. Modena 51(2), 415-431 (2003)

12. Brooks, J.K., Candeloro, D.: The Rickart integral and Yosida-Hewitt decompositions. Tatra Mt. Math. Publ. 28, 227-240 (2004)

13. Brooks, J.K., Candeloro, D.: On the space of functions integrable with respect to functions of unbounded variation. Atti Sem. Mat. Fis. Univ. Modena 54(1-2), 115-123 (2006)

14. Candeloro, D.: Riemann-Stieltjes integration in Riesz spaces. Rend. Math. Appl. 16(4), 563-585 (1996)

15. Candeloro, D.: L'integrale di Burkill-Cesari e le sue relazioni con continuità assoluta. Rend. Circ. Mat. Palermo 26(1-3), 251-274 (1977)

16. Candeloro, D., Croitoru, A., Gavrilut, A., Iosif, A., Sambucini, A.R.: Properties of the Riemann-Lebesgue integrability in the non-additive case. Rend. Circ. Mat. Palermo (2019). https://doi.org/10.1007/s12215019-00419-y

17. Candeloro, D., Croitoru, A., Gavrilut, A., Sambucini, A.R.: An extension of the Birkhoff integrability for multifunctions. Mediterr. J. Math. 13(5), 2551-2575 (2016)

18. Candeloro, D., Croitoru, A., Gavrilut, A., Sambucini, A.R.: Atomicity related to non-additive integrability. Rend. Circ. Mat. Palermo 65(3), 435-449 (2016)

19. Candeloro, D., Croitoru, A., Gavrilut, A., Sambucini, A.R.: A multivalued version of the Radon-Nikodým theorem. Aust. J. Math. Anal. Appl. 15(2), 1-16 (2018)

20. Candeloro, D., Di Piazza, L., Musiał, K., Sambucini, A.R.: Gauge integrals and selections of weakly compact valued multifunctions. J. Math. Anal. Appl. 441(1), 293-308 (2016)

21. Candeloro, D., Di Piazza, L., Musiał, K., Sambucini, A.R.: Relations among gauge and Pettis for cwk(X)valued multifunctions. Ann. Mat. Pura Appl. 197(1), 171-183 (2018)

22. Candeloro, D., Di Piazza, L., Musiał, K., Sambucini, A.R.: Some new results on integration for multifunctions. Ric. Mat. 67(2), 361-372 (2018)

23. Candeloro, D., Di Piazza, L., Musiał, K., Sambucini, A.R.: Multifunctions determined by integrable functions. Int. J. Approx. Reason. 112, 140-148 (2019)

24. Candeloro, D., Mesiar, R., Sambucini, A.R.: A special class of fuzzy measures: Choquet integral and applications. Fuzzy Sets Syst. 355, 83-99 (2019) 
25. Candeloro, D., Sambucini, A. R., Trastulli, L.: A vector Girsanov result and its application to conditional measures via the Birkhoff integrability. Mediterr. J. Math. 16(6), 144 (2019). https://doi.org/10.1007/ s00009-019-1431-x

26. Riesz, F.: Sur certains systèmes singuliers d'équations intégrales. Ann. Sci. Ecole Norm. Sup. Paris 28, 33-68 (1911)

27. Vainikko, G.: Cordial Volterra integral equations 1. Num. Funct. Anal. Optim. 30, 1145-1172 (2009)

28. Vainikko, G.: Cordial Volterra integral equations 2. Num. Funct. Anal. Optim. 31, 191-219 (2010)

29. Vainikko, G.: Cordial Volterra integral operators in spaces $L^{p}(0, T)$. J. Integr. Equations Appl. 31(2), 283-305 (2019)

30. Waterman, D.: On $\Lambda$-bounded variation. Studia Math. 57, 33-45 (1976)

31. Waterman, D.: Fourier series of functions of $\Lambda$-bounded variation. Proc. Am. Math. Soc. 74(1), 119-123 (1979)

32. Wiener, N.: The quadratic variation of a function and its Fourier coefficients. J. Math. Phys. MIT 3, 73-94 (1924)

Publisher's Note Springer Nature remains neutral with regard to jurisdictional claims in published maps and institutional affiliations. 